\title{
Evaluation of Digitizer Properties on Pulse Shape Discrimination in a gelled NE213 Cell.
}

\author{
Mark A. Nelson, Derek R. Dinwiddie, William Baird, and Brian D. Rooney
}

\begin{abstract}
The figure-of-merit for a gelled NE213 scintillator cell from Bubble Technology Industries is determined for a variety of digitizing speeds and vertical resolutions using the digital-charge-integration-comparison method for pulse-shapediscrimination. If a digitizing board allows for an increase in the vertical resolution at the expense of the digitization rate, then there is an optimal combination of the two. For the Gage Board 82G, a board that does allow this tradeoff to be made, the best pulse shape discrimination occurred for digitization rates and vertical resolutions of $1 \mathrm{GS} / \mathrm{s}$ and 8 bits, $500 \mathrm{MS} / \mathrm{s}$ and 9 bits, and $250 \mathrm{MS} / \mathrm{s}$ with 9 bits.
\end{abstract}

Index Terms-NE213, BC501A, EJ301A, digital charge integration comparison, pulse shape discrimination, fast-neutron detection.

\section{INTRODUCTION}

The liquid scintillator NE213 is popular for fast-neutron detection. In order to discriminate gamma-induced events from neutron-induced events occurring in the scintillator, pulseshape-discrimination (PSD) is performed. Pulse-shapediscrimination can be performed because the light-output in the scintillator from gamma-ray interactions decays at a different rate than from neutron interactions. There are a variety of algorithms for PSD, including the chargeintegration-comparison (CIC) method [1]. In this method, interactions in the scintillator are discriminated by examining the rising edge of the integrated light output for each interaction and comparing it with the total light output.

In order to collect the light from the scintillator, the scintillator needs to be mated with a light collection device, which is commonly a photo-multiplier-tube (PMT). When the pulses from the PMT are integrated, the pulse from a gammaray interaction rises much faster than a pulse due to a neutron interaction. The CIC method integrates a region on the leading edge of a pulse from a charge-integration preamplifier and

Manuscript received October 16, 2004. This work was supported in part by the U.S. Department of Energy.

Mark A. Nelson is with the Los Alamos National Laboratory, Los Alamos, NM 87545 USA (phone: 505-984-8626; fax: ; email: manenlson@lanl.gov).

Derek R. Dinwiddie is with the Los Alamos National Laboratory, Los Alamos, NM 87545 USA (email: derekd@lanl.gov).

William Baird is with the Los Alamos National Laboratory, Los Alamos, NM 87545 USA (email: baird@lanl.gov).

Brian D Rooney is with the Los Alamos National Laboratory, Los Alamos, NM 87545 USA (email: rooney@lanl.gov ). compares it to the pulse height. When the region on the leading edge is correctly set, a clear delineation between the gammainteractions and neutron-interactions in the scintillation cell are obvious when the ratio of the pulse height to pulse-shape number is plotted in a histogram.

The digital-charge-integration-comparison (DCIC) method digitizes the integrated pulse from the preamplifier and then adds up a selected region of the rising edge of the integrated pulse and compares it with the pulse height. This technique is computationally fast and simple. However, the accuracy of identifying neutron pulses is dependent upon the sampling rate and the vertical resolution of the digitizer. Recent trends when using this technique is to use faster digitizers with some sampling rates up to $5 \mathrm{GS} / \mathrm{s}$ [2]. However, various experiments have reported good discrimination with digitizing speeds less than $1 \mathrm{GS} / \mathrm{s}$ and it has been suggested that digitizing the signal with a finer vertical resolution may yield better PSD than a sampling at a faster rate [3].

Current digitizing boards can sample in the range of a $\mathrm{GS} / \mathrm{s}$ with 8-bit resolution but higher vertical-resolution digitizers are approximately an order of magnitude slower. Some of these boards can also trade sampling rate for a better sampling resolution. This ability of these digitizing boards affords us the ability to study the effects that the digitizing rate and the vertical resolution have on PSD in NE213.

\section{EXPERIMENTAL SETUP}

A ${ }^{252} \mathrm{Cf}$ source was placed in front of a $7.6 \times 7.6 \mathrm{~cm}$ gelled NE213 liquid scintillator cell from Bubble Technology Industries that was connected to a PMT (Electron Tubes 9273) with an associated base. The PMT base was connected to an Ortec model 113 preamp and the resulting pulses were digitized at various sampling rates with a Gage $82 \mathrm{G}$ digitizing board set at a range of \pm 5 Volts. Additionally, a constant fraction discriminator (Ortec 473A) was used to trigger the digitizer. This setup allowed for the analysis of neutrons in the energy range of 0.8 to $3 \mathrm{MeV}$ and a schematic of the setup is displayed in Fig. 1.

The $82 \mathrm{G}$ was installed in a personal computer and operated with the digitizing software GageScope ${ }^{\circledR}$. GageScope ${ }^{\circledR}$ allows for the digitizing board to operate at different vertical resolutions at the expense of digitizing speed. The maximum digitizing speed of the $82 \mathrm{G}$ is $2 \mathrm{GS} / \mathrm{s}$ with a vertical resolution of 8 bits. When the vertical resolution is increased by 1 bit, the 
maximum sampling rate is reduced by a factor of two. For example, the maximum sampling rate when sampling at 9 bits is $1 \mathrm{GS} / \mathrm{s}$, at $10 \mathrm{bits}$ it is $500 \mathrm{MS} / \mathrm{s}$, and so on.

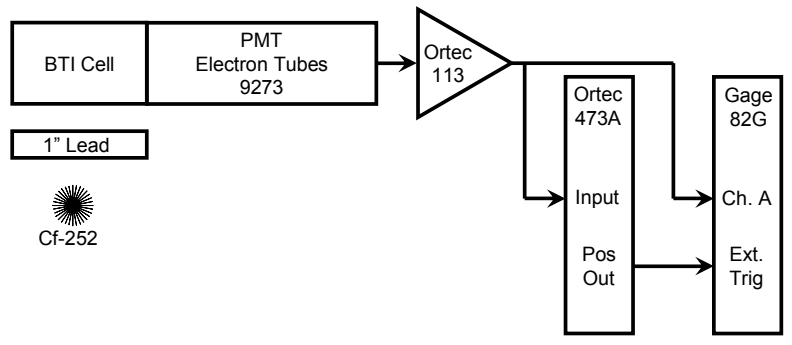

Fig. 1. Schematic of experimental setup.

The $82 \mathrm{G}$ can digitize signals with a vertical resolution of 8 , $9,10,11,12$, and 13 bits with various maximum sampling rates. Unfortunately, the vertical offset of the signals coming into the digitizing board could not be changed from $0 \mathrm{~V}$ and the digitizing board is set to sample both the positive and negative potential of signals. This limitation reduced the effective vertical resolution by one. Hence, the effective vertical resolutions were $7,8,9,10,11$, and 12 bits with maximum sampling rate of $2 \mathrm{GS} / \mathrm{s}, 1 \mathrm{GS} / \mathrm{s}, 500 \mathrm{MS} / \mathrm{s}, 250$ $\mathrm{MS} / \mathrm{s}, 125 \mathrm{Ms} / \mathrm{s}$, and $62.5 \mathrm{MS} / \mathrm{s}$ respectively.

\section{DATA ANALYSIS}

For each sampling set, 20,000 waveforms were saved to a hard-drive and postanalyzed to determine the pulse height and a pulse-shape number. To determine the pulse height, a region was set that began from the pulse start and the values of the digitized pulse were summed up for a specific amount of time after the pulse start. A second region used to determine a shape value summed up values on the leading edge of the digitized pulse. The start of this region was delayed slightly from the pulse start and was much shorter in duration than the region used for height determination.

The height-to-shape ratio was then plotted in a histogram and if the integration regions are correctly defined, two prominent peaks were present. One peak corresponded to the gamma-ray induced events and the other to neutron induced events. From the histogram, the figure of merit (FoM) was determined. The figure of merit value [4] corresponds to the relative effectiveness of discrimination. The larger the FoM the better the PSD and the equation for the FoM value is

$$
\mathrm{FoM}=\frac{\text { Peak }_{n}-\text { Peak }_{\gamma}}{\left(\mathrm{FWHM}_{n}+\mathrm{FWHM}_{\gamma}\right)} .
$$

In addition to analyzing the original sample sets, some of the saved sample sets were down-sampled in the time regime by a factor of two. For example, if a series of pulses were sampled at $2 \mathrm{GS} / \mathrm{s}$ at 7 bits, every other point in the saved waveforms were omitted to yield a separate sample set equivalent to a sample set digitized at $1 \mathrm{GS} / \mathrm{s}$ and 7 bits. This down-sampling process was sometimes repeated more than once and allowed more data sets to be analyzed with different time scales.

The quality of PSD when using DCIC is highly susceptible to the boundaries of the integration regions, especially the region that determines the shape-number of the pulse. To expedite the analysis, a computer program was written that varied the start and stop of the integration region that determined the pulse shape number, varied the ending value that determines the pulse height, and determined the pulse height-to-shape ratio for each sampling set. After the pulse height-to-shape ratios for each pulse was determined, another computer program plotted the ratios in a histogram and fit two Gaussian curves to the resulting graph. The FoM was determined from the values in the Gaussian fit and the largest FoM value was then recorded.

The integration regions for the pulse height all started at 0 $\mu \mathrm{s}$ and lasted from $6 \mu \mathrm{s}$ to $8 \mu \mathrm{s}$. The total time for the integration region for the pulse-height number ranged from 48 to $80 \mathrm{~ns}$, depending upon the digitization rate. The estimated error was estimated to be $5 \%$ and was determined by repeating the analysis of different sampling sets and taking the standard deviation.

\section{EXPERIMENTAL RESULTS}

The FoM values as a function of digitizing speed and resolution are listed in Table 1. The best pulse separation in this table occurs at sampling rates and vertical resolutions of 1 $\mathrm{GS} / \mathrm{s}$ and 8 bits, $500 \mathrm{MS} / \mathrm{s}$ and 9 bits, and $250 \mathrm{MS} / \mathrm{s}$ and 9 bits with FoM values of $1.39,1.38$, and 1.38 respectively. Because the error associated with these three measurements is $5 \%$, they are equal.

TABLE 1

THE AVERAGE FigurE OF MERIT (FOM) AS A FUNCTION OF DIGITIZATION RATE AND SAMPLING. THE PERCENT ERROR FOR EACH VALUE IS ESTIMATED TO BE $5 \%$.

\begin{tabular}{ccccccc}
\hline \hline $\begin{array}{c}\text { Sampling } \\
\text { Rate }\end{array}$ & 7 & 8 & 9 & 10 & 11 & 12 \\
\hline $2 \mathrm{GS} / \mathrm{s}$ & 1.29 & & & & & \\
$1 \mathrm{GS} / \mathrm{s}$ & 1.19 & 1.39 & & & & \\
$500 \mathrm{MS} / \mathrm{s}$ & 1.17 & 1.25 & 1.38 & & & \\
$250 \mathrm{MS} / \mathrm{s}$ & 1.03 & 1.23 & 1.38 & 1.32 & & \\
$125 \mathrm{MS} / \mathrm{s}$ & & 1.17 & 1.31 & 1.27 & 1.30 & \\
$62.5 \mathrm{MS} / \mathrm{s}$ & & 1.01 & 1.02 & 1.12 & 1.16 & 1.15 \\
\hline \hline
\end{tabular}

The FoM as a function of sampling rate for different resolutions is plotted in Fig. 2 and the FoM as a function of vertical resolution for the different digitizing speeds if plotted in Fig. 3. The general trend for Fig. 2 is for the FoM to increase as the sampling rate increases and similarly in Fig. 3, the FoM increases at the resolution increases. 


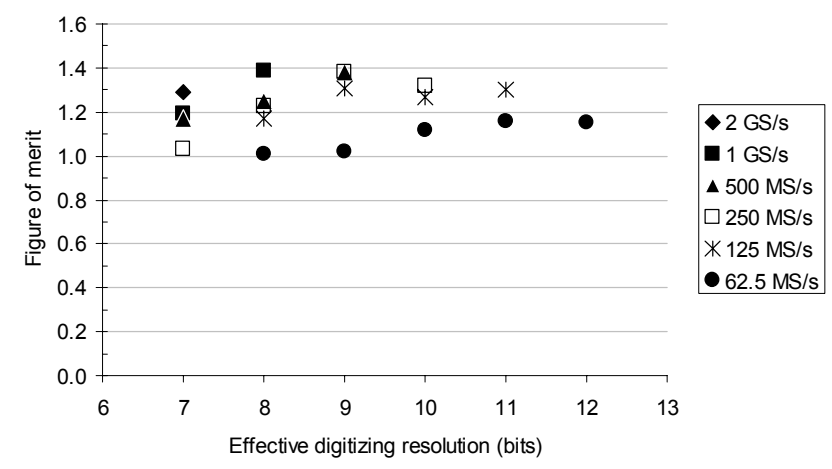

Fig. 2. The figure of merit as a function of vertical resolution for multiple sampling rates.

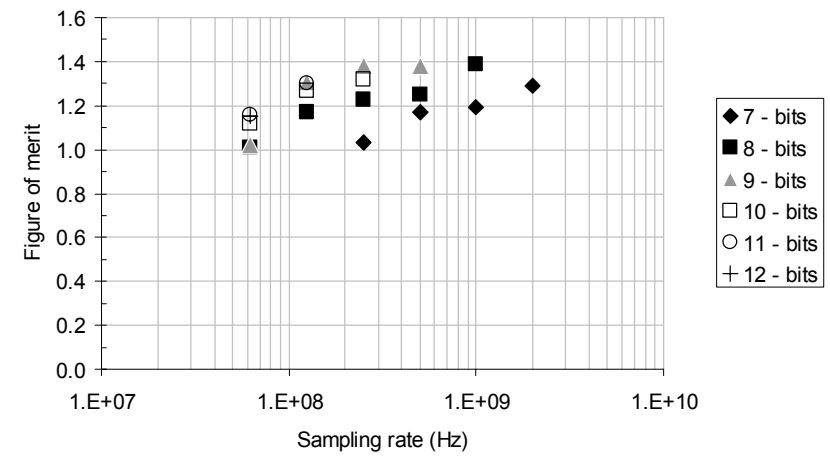

Fig. 3. The figure of merit as a function of sampling rate for multiple vertical resolution.

Fig. 4 is a plot of the FoM as a function of resolution and digitization speed. This graph shows the FoM when you trade digitization speed for vertical resolution when using the $82 \mathrm{G}$ digitizing board at its maximum limits. Fig. 5 is a similar plot except it is for when the $82 \mathrm{G}$ is operated in a state one less than its maximum limits. These data points can be found in the diagonals in Table 1. For Fig. 4 these points are shown in the diagonal that starts at the $2 \mathrm{GS} / \mathrm{s}$ and 7 bit intersection and for Fig. 5 these start at the $1 \mathrm{GS} / \mathrm{s}$ and 7 bit intersection. Both Fig. 4 and Fig. 5 show that if one can trade resolution for speed that there is an optimal combination of vertical resolution and digitizing speed.

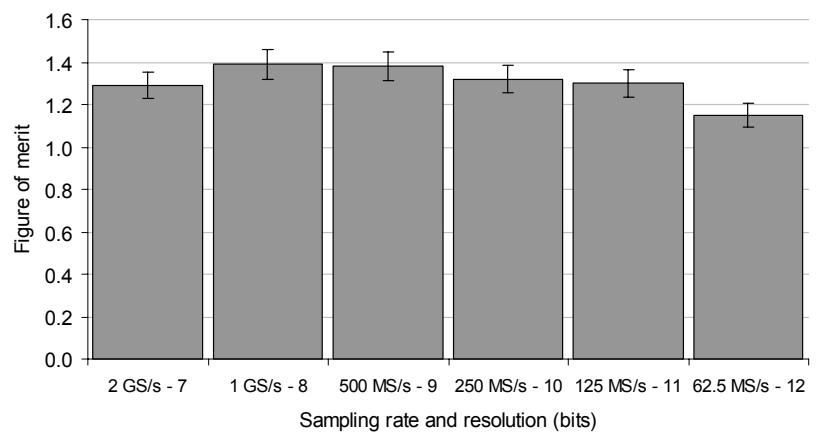

Fig. 4. The figure of merit for the $82 \mathrm{G}$ with a maximum sampling rate of 2 $\mathrm{GS} / \mathrm{s}$ at 7-bits. The other data points are when the sampling rate is reduced in exchange for an increase in vertical resolution.

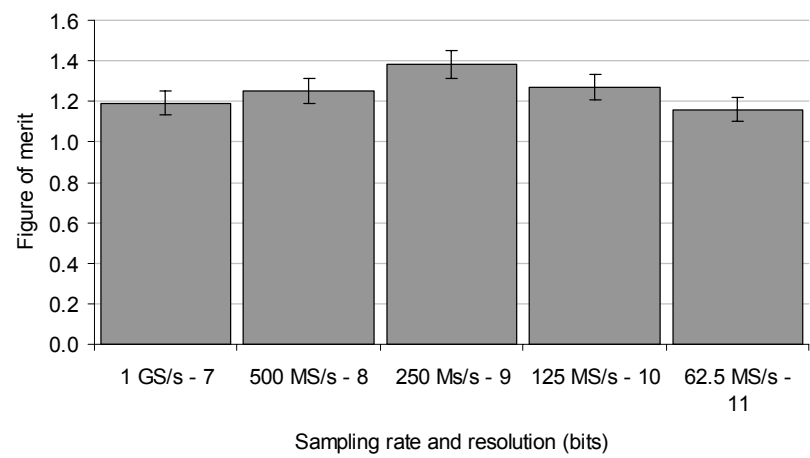

Fig. 5. The figure of merit for the $82 \mathrm{G}$ with a maximum sampling rate of 1 $\mathrm{GS} / \mathrm{s}$ at 7-bits. The other data points are when the sampling rate is reduced in exchange for an increase in vertical resolution.

\section{CONCLUSION}

The recent trend in implementing the DCIC method is to increase the sampling speed while not increasing the vertical resolution. This is not the only way to increase the quality of PSD. Improvements in PSD can also be achieved by increasing vertical resolution. This paper shows that if you're able to trade sampling rate for an increase in the vertical resolution that this may yield a better FoM.

\section{REFERENCES}

[1] D. Wolski, M. Moszyński, T. Ludziejewski, A. Johnson, W. Klamra, and Ö. Skeppstedt, "Comparison of $\mathrm{n}$-g discrimination by zero-crossing and digital charge comparison Methods", Nucl. Inst. and Meth. A, vol. 360,1995 , pp. 584-592.

[2] T. Kurahashi, H. Takahashi, E. Takada, M. Nakazawa, and T. Iguchi, "A Digital Waveform Discrimination System for an NE213 Scintillator using a VXIbus General Purpose Waveform Digitizer", Conf. Rec. of 1996 IEEE Nucl. Sci. Symp., Anaheim, CA 1996, pp. 457-459.

[3] V. Jordanov, and G. Knoll, "Digital Pulse-Shape Analyzer Based on Fast Sampling of an Integrated Charge Pulse", IEEE Trans. Nuc. Sci., vol. 42, August 1995, pp. 683-687.

[4] G. Knoll, Radiation Detection and Measurements $2^{\text {nd }}$ Edition. New York: Wiley and Sons, 1989, pp. 646. 